\title{
Ictiofauna das praias de Cabuçu e Berlinque: Uma contribuição ao conhecimento das comunidades de peixes na Baía de Todos os Santos - Bahia - Brasil
}

\author{
Jailza Tavares de Oliveira-Silva ${ }^{1}$ \\ Marlene Campos Peso-Aguiar ${ }^{2}$ \\ Paulo Roberto Duarte Lopes ${ }^{*}$ \\ ${ }^{1}$ Laboratório de Ictiologia, Departamento de Ciências Biológicas \\ Universidade Estadual de Feira de Santana, Campus Universitário, km 03, BR116 \\ CEP 44031-460, Feira de Santana, - BA, Brasil \\ jtosilva@yahoo.com.br \\ ${ }^{2}$ Instituto de Biologia, Universidade Federal da Bahia, Salvador - BA, Brasil \\ mpeso@ufba.br \\ *Autor para correspondência \\ andarilho40@yahoo.com.br
}

Submetido em 06/09/2007 Aceito para publicação em 13/06/2008

\section{Resumo}

Este trabalho apresenta a composição da ictiofauna e a análise espacial e sazonal da estrutura da comunidade

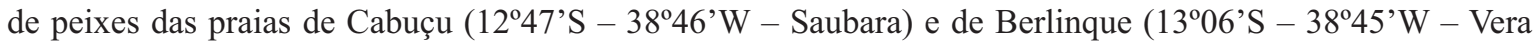
Cruz), Baía de Todos os Santos $\left(13^{\circ} \mathrm{S}-38^{\circ} \mathrm{W}\right)$, estado da Bahia, Brasil. Os peixes foram coletados entre julho de 2002 e julho de 2003, durante a baixa-mar de marés de sizígia. Fatores abióticos, como pH, temperatura da água e salinidade, foram mensurados. A similaridade entre os locais de coleta foi analisada através da análise de Cluster, baseada sobre uma matriz de dados de presença-ausência das espécies, utilizando-se o coeficiente de Sorensen. Um total de 719 peixes e $2231,77 \mathrm{~g}$ de 63 espécies na praia de Cabuçu sendo que Lutjanus synagris, Larimus breviceps e Chaetodipterus faber dominaram. Na praia de Berlinque, foram coletados 381 peixes e 2605,64g, de 40 espécies. Polydactylus virginicus, Ophioscion punctatissimus e Conodon nobilis foram as espécies dominantes. Nenhuma tendência sazonal foi observada nos fatores abióticos, abundância e índices de comunidades de peixes nas duas praias. As rotinas ANOSIM e SIMPER do Primer demonstram que a composição ictiofaunística nas duas praias diferiu significativamente.

Unitermos: ictiofauna, praia de Cabuçu, praia de Berlinque, Baía de Todos os Santos, Bahia

\section{Abstract}

Ichthyofauna of Cabuçu and Berlinque beaches: A comparative analysis of fish communities in Todos os Santos Bay, Bahia - Brazil. This study presents the composition of the ichthyofauna and the spatial and seasonal analysis of fish community structure at Cabuçu (12 $47^{\prime} \mathrm{S}-38^{\circ} 46^{\prime} \mathrm{W}$ - Saubara) and Berlinque beaches $\left(13^{\circ} 06^{\prime} \mathrm{S}-38^{\circ} 45^{\prime} \mathrm{W}\right.$ - Vera Cruz) in Todos os Santos Bay $\left(13^{\circ} \mathrm{S}-38^{\circ} \mathrm{W}\right)$, Bahia State, Brazil. Fish were collected between July 2002 and July 2003, during the lowest of the syzygial tides. Abiotic factors such as the $\mathrm{pH}$, temperature and salinity of the water were measured. The similarity among the sampling sites was 
analyzed by cluster analysis based on a presence-absence data matrix of species using the Sorensen coefficient. A total of 719 fish (63 species) and 2,231.77g were captured at Cabuçu beach. At Berlinque beach, a total of 381 fish (40 species) and 2,605.64g were captured. Community indexes do not indicate seasonal changes in the fish communities of the two beaches. The ANOSIM and SIMPER routines of the Primer software demonstrate that the ichthyofaunal composition of the two beaches differs markedly.

Key words: ichthyofauna, Cabuçu beach, Berlinque beach, Todos os Santos Bay, Bahia

\section{Introdução}

Ambientes costeiros, como estuários, lagoas e baías são essenciais ao desenvolvimento dos recursos pesqueiros de importância econômica e ambiental, tendo em vista o seu papel no desempenho dos ciclos biológicos, atuando como berçários, tanto de espécies características desses ambientes como de outras marinhas que migram para essas áreas durante a fase reprodutiva. Fauna e flora associadas a esses ecossistemas constituem significativa fonte de alimentos para as populações humanas, onde os peixes desempenham um dos papéis mais significativos no balanço energético desses ecossistemas (RodriguezRomero et al., 1994; Santos, 2001).

As praias arenosas representam o mais amplo dos ecossistemas sedimentares costeiros. Como nos manguezais e regiões estuarinas, os efeitos da maré servem para distribuir nutrientes e minerais através do ecossistema, essencial no caso das praias arenosas, considerando que a produtividade primária "in situ" é pouco significativa (Teixeira e Almeida, 1998).

A comunidade ictiofaunística da zona de arrebentação de praias arenosas é dinâmica, com pequeno número de espécies dominantes e residentes e com variação sazonal na abundância (Giannini e Paiva Filho, 1995).

Apesar do valor significativo do papel desses ecossistemas para vários organismos aquáticos, ainda não existem estudos sobre a avaliação da composição quali-quantitativa da ictiofauna nessas regiões.

Paiva-Filho e Toscano (1987) analisaram a ictiofauna na zona de entre-marés em duas praias, de substrato arenoso e lamosa, entre maio de 1984 e maio de 1985, em São Vicente (estado de São Paulo), e observaram diferenças na composição das famílias de peixes capturadas.
Devido à existência de grande diversidade de ambientes estuarinos-lagunares costeiros na Baía de Todos os Santos (BTS), como manguezais, restingas, etc (Falcón, 1997), e considerando as poucas informações disponíveis sobre esses ecossistemas com relação à estrutura da ictiofauna existente, este estudo objetiva caracterizar e comparar a ictiofauna de duas praias de substratos distintos, lamoso (Cabuçu) e arenoso (Berlinque), estado da Bahia (litoral nordeste do Brasil), identificando as possíveis diferenças através de uma análise espacial e temporal destas comunidades costeiras e suas interações com o meio físico.

\section{Material e Métodos}

A Praia de Cabuçu, localizada em $12^{\circ} 47^{\prime} \mathrm{S}-$ $38^{\circ} 46^{\prime} \mathrm{W}$, no município de Saubara, região ocidental da BTS, apresenta substrato do tipo lamoso (Figura 1).

A Praia de Berlinque $\left(13^{\circ} 06^{\prime} \mathrm{S}-38^{\circ} 45^{\prime} \mathrm{W}\right)$ é uma praia exposta, constituída de substrato arenoso, localizada no lado oriental da Ilha de Itaparica (município de Vera Cruz) (Figura 1). Ambas as praias são importantes locais para a prática esportiva e pesca artesanal, no entanto estão sob a pressão decorrente da especulação imobiliária impulsionada pelo turismo.

As amostragens foram realizadas bimestralmente no período de julho/2002 a julho/2003, durante a baixa-mar das marés de sizígia, com rede de arrasto manual medindo $7,02 \mathrm{~m}$ de comprimento, $10,31 \mathrm{~m}$ de abertura, $1,78 \mathrm{~m}$ de altura e malha de $2,0 \mathrm{~cm}$ entre nós. Em cada praia foram efetuados 5 arrastos de $100 \mathrm{~m}$ de extensão, totalizando $500 \mathrm{~m}$, paralelos à linha da costa, em profundidade máxima de cerca de $1,70 \mathrm{~m}$, durante o período diurno. 


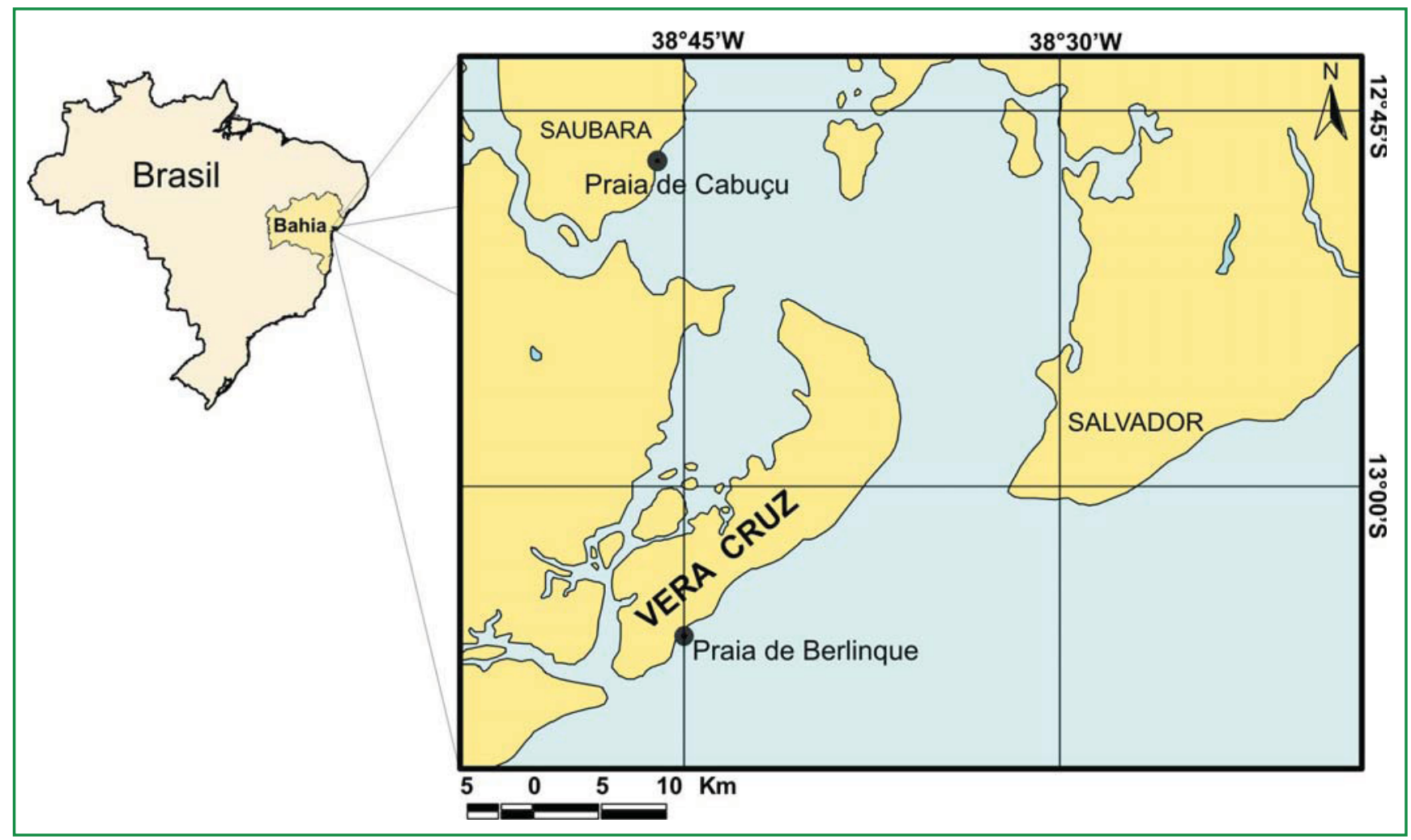

FIGURA 1: Mapa indicando os locais de coleta.

Foram anotados durante as amostragens, em arrastos alternados, os seguintes parâmetros ambientais: temperatura da água (medido com termômetro de mercúrio com precisão de $\left.1,0^{\circ} \mathrm{C}\right), \mathrm{pH}(\mathrm{pHmetro}$ de bancada com precisão de 0,01 ) e salinidade (refratômetro óptico). Os dados meteorológicos de pluviosidade, nos meses de coleta, foram fornecidos pelo Instituto Nacional de Meteorologia da Bahia (INMET) e correspondem a valores médios mensais calculados para a estação 83229 - Salvador (1301'00”S - 038 $\left.31^{\circ} 00^{\prime \prime} \mathrm{W}\right)$.

Os exemplares de peixes capturados em cada praia foram identificados ao menor nível taxonômico possível sendo realizada a biometria dos espécimes para a obtenção do comprimento total ( $\mathrm{mm})$ e do peso (g). Material testemunho encontra-se depositado na coleção do Laboratório de Ictiologia (Departamento de Ciências Biológicas) da Universidade Estadual de Feira de Santana (Bahia) conservado em álcool 70\%.

\section{Análise dos dados}

Devido à inexistência de estações climáticas bem definidas na região nordeste, foi consultado um gráfico de balanço hídrico, em ciclo de 30 anos, da BTS e a partir dos dados fornecidos pelo INMET, os meses amostrados foram agrupados em estação chuvosa (julho de 2002 e 2003, setembro de 2002, março e maio de 2003) e estação seca (novembro de 2002 e janeiro de 2003).

A constância das espécies foi calculada em função do número de vezes que cada espécie ocorreu em relação ao total de coletas realizadas (Dajoz, 1973).

Para identificar as espécies dominantes em cada localidade, foi utilizado o Índice Ponderal ou Índice de Importância Relativa definido por Beaumord (1991).

$$
I I R=\frac{(\mathrm{NiPiC})}{\sum(\mathrm{NiPiC})} \times 100
$$

onde:

$\mathrm{Ni}=$ número de exemplares da espécie i;

$\mathrm{Pi}=$ peso dos exemplares da espécie i e

$\mathrm{C}=$ constância ou freqüência de ocorrência. 
Foram usados os seguintes índices de diversidade: Riqueza de Espécies de Margalef, Índice de Diversidade de Shannon-Wiener e Equitabilidade.

A análise de variância não paramétrica de KruskalWallis $(\alpha<0,05)$ foi utilizada na análise das séries temporais de dados ambientais durante o período do estudo, seguida dos testes de comparações múltiplas de Dunn. Procedimento similar foi utilizado na comparação entre os dados biológicos. Também foi utilizado o teste de Mann-Whitney $(\alpha<0,05)$ na averiguação dos dados abióticos entre as praias estudadas (Zar, 1999). As análises estatísticas foram realizadas através do programa INSTAT.

A análise de grupamentos das amostras e o mapeamento multidimensional da similaridade dos grupos (non Metric Multidimensional Scaling nMDS) foram realizados visando avaliar a distância ecológica qualitativa entre as estações investigadas ao longo do tempo. Para tal, foram construídas matrizes de similaridade (presença $\mathrm{x}$ ausência) utilizando-se o coeficiente de similaridade de Sorensen (modo Q).

A similaridade das percentagens (SIMPER) foi utilizada para identificar as espécies responsáveis principais pelos índices de dissimilaridade dentro de cada grupo. A avaliação da similaridade ictiofaunística entre as duas localidades utilizou o teste ANOSIM (análise univariada de similaridade), usando como medida de similaridade o índice de Bray-Curtis. A análise BIOENV foi utilizada para relacionar os dados bióticos e ambientais, indicando os parâmetros mais importantes na estruturação do padrão de distribuição temporal observado na composição e abundância das espécies. As análises foram realizadas através do programa estatístico PRIMER 5 for Windows (Clarke e Warwick, 2001).

\section{Resultados}

\section{Parâmetros abióticos}

Na praia de Cabuçu, a temperatura da água variou entre $25,6^{\circ} \mathrm{C}$ e $31^{\circ} \mathrm{C}$. Foram significativas as diferenças entre os meses de janeiro e julho/03, enquanto que na praia de Berlinque, a temperatura variou entre $25^{\circ} \mathrm{C}$ e $31^{\circ} \mathrm{C}$, com diferenças significativas entre os de julho/2002 e janeiro/2003, julho de 2002 e março de 2003, março e maio/2003. As médias mais altas da temperatura ocorreram em janeiro, março e novembro em ambas as praias. Não foram verificadas diferenças significativas para a temperatura da água entre as praias estudadas (Figura 2).

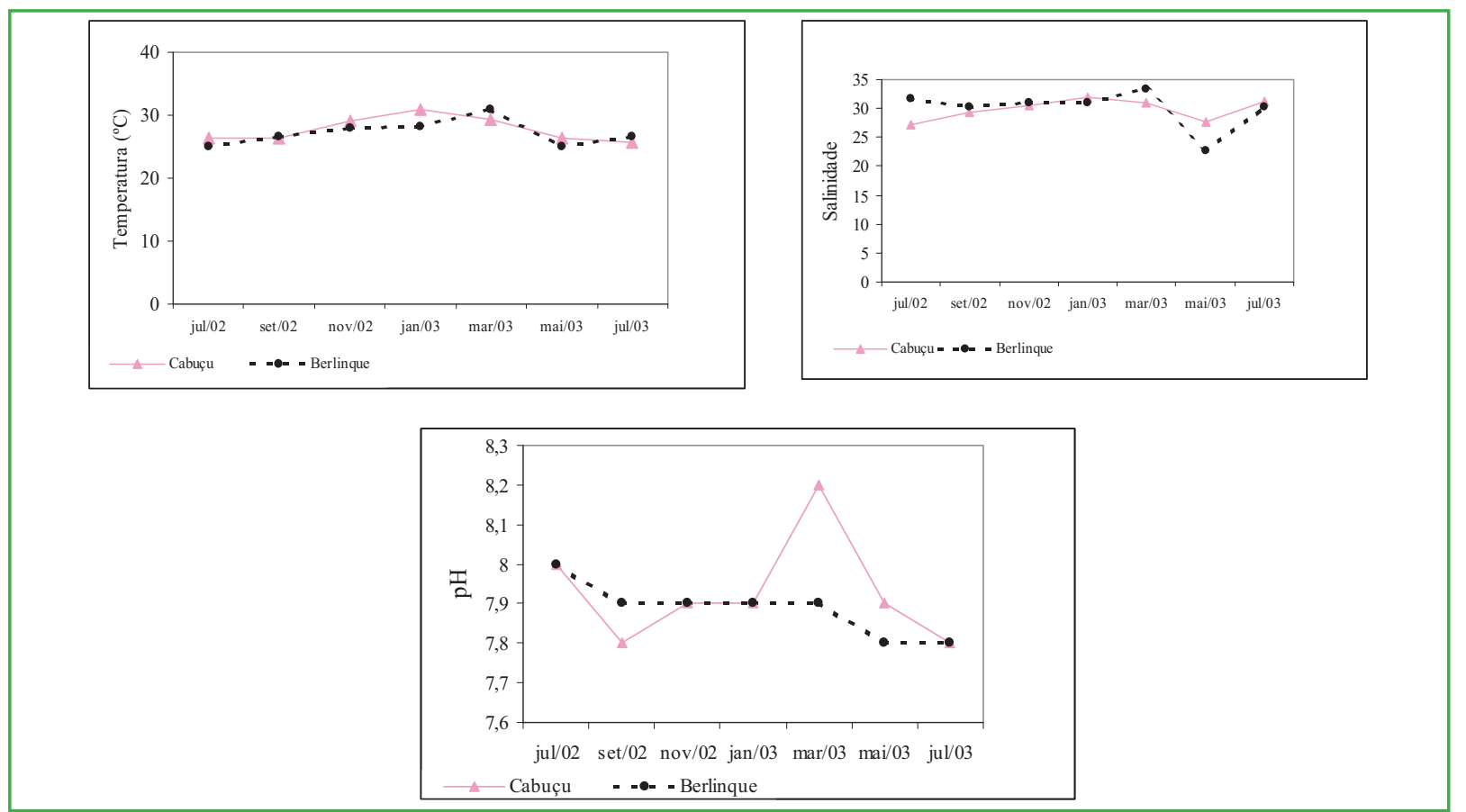

FIGURA 2: Variação mensal dos valores da temperatura, salinidade e $\mathrm{pH}$ registrados nas praias de Cabuçu e Berlinque, no período de julho/02 a julho/03. 
A salinidade variou entre 27,2 e 32 na praia de Cabuçu com diferenças significativas entre os meses de julho/2002 e janeiro/2003, e em Berlinque a salinidade variou entre 22,6 a 33,3 apresentando diferenças temporais significativas entre julho/2002 e maio/2003 e março e maio de 2003. Todavia, não houve diferenças significativas deste parâmetro entre as praias estudadas (Figura 2).

$\mathrm{O}$ pH variou entre 7,8 a 8,2 em Cabuçu, e 7,8 a 8 em Berlinque. Nenhuma diferença foi encontrada entre os meses de amostragens nas praias estudadas como também não houve diferenças espaciais quanto a este parâmetro (Figura 2).

\section{Ictiofauna}

Foram capturados, na praia de Cabuçu, 719 indivíduos e $2231,77 \mathrm{~g}$, pertencentes a 63 espécies, 52 gêneros, 29 famílias (com predominância de espécies das famílias Sciaenidae, Carangidae, Gerreidae e Engraulidae) e 12 ordens (Tabela 1). Na praia de

TABELA 1: Lista de espécies, número de indivíduos (n), participação percentual do peso (p \%), constância (C) e índice de importância relativa (IIR) das espécies amostradas nas praias de Cabuçu (Saubara) e Berlinque (Vera Cruz) entre julho de 2002 e julho de 2003.

\begin{tabular}{|c|c|c|c|c|c|c|c|c|c|c|}
\hline \multirow{2}{*}{ Espécies } & \multicolumn{5}{|c|}{ Cabuçu } & \multicolumn{5}{|c|}{ Berlinque } \\
\hline & $\mathbf{n}$ & $\%(n)$ & p (\%) & C & IIR & $\mathrm{n}$ & $\%(n)$ & p (\%) & $\mathrm{C}$ & IIR \\
\hline Albula vulpes & 8 & 1,11 & 0,6 & Ac & 0,29 & 2 & 0,5 & 1,04 & A & 0,03 \\
\hline Anchoa sp. & 66 & 9,2 & 0,52 & $\mathrm{C}$ & 3,16 & 26 & 6,82 & 0,54 & $\mathrm{C}$ & 0,92 \\
\hline Anchovia clupeoides & 1 & 0,14 & 0,09 & A & $<0,01$ & 2 & 0,5 & 0,48 & A & 0,01 \\
\hline Cetengraulis edentulus & 7 & 0,98 & 7,1 & $\mathrm{C}$ & 3,80 & 1 & 0,26 & 1,61 & A & 0,02 \\
\hline Engraulis anchoita & & & & & & 8 & 2,10 & 0,3 & Ac & 0,11 \\
\hline Lycengraulis grossidens & 2 & 0,28 & 1,94 & A & 0,05 & 7 & 1,84 & 6,49 & $\mathrm{C}$ & 2,93 \\
\hline Synodus foetens & 1 & 0,14 & 0,18 & A & $<0,01$ & & & & & \\
\hline Cathorops spixii & & & & & & 1 & 0,26 & 0,15 & A & $<0,01$ \\
\hline Thalossophryne nattereri & 10 & 1,39 & 4,72 & $\mathrm{C}$ & 3,63 & & & & & \\
\hline Ogcocephalus vespertilio & 1 & 0,14 & 0,53 & A & $<0,01$ & & & & & \\
\hline Mugil sp & 6 & 0,83 & 0,7 & Ac & 0,18 & & & & & \\
\hline Atherinella blackburni & & & & & & 3 & 0,78 & 0,14 & Ac & 0,02 \\
\hline Atherinella brasiliensis & 34 & 4,72 & 1 & $\mathrm{C}$ & 2,10 & & & & & \\
\hline Hippocampus reidi & 3 & 0,41 & 0,69 & A & 0,03 & 1 & 0,26 & 0,26 & A & $<0,01$ \\
\hline Syngnathus sp. & 9 & 1,25 & 0,07 & $\mathrm{Ac}$ & 0,02 & & & & & \\
\hline Fistularia petimba & 3 & 0,42 & 0,65 & $\mathrm{Ac}$ & 0,02 & & & & & \\
\hline Fistularia tabacaria & 1 & 0,14 & 1,34 & A & 0,04 & & & & & \\
\hline Prionotus punctatus & 13 & 1,80 & 0,7 & Ac & 0,4 & & & & & \\
\hline Centropomus undecimalis & 2 & 0,28 & 2,26 & $\mathrm{~A}$ & 0,06 & & & & & \\
\hline Centropomus parallelus & 1 & 0,14 & 1,13 & A & 0,01 & & & & & \\
\hline Rypticus randalli & 11 & 1,53 & 3,97 & $\mathrm{C}$ & 2,69 & 1 & 0,26 & 0,1 & A & $<0,01$ \\
\hline Serranus flaviventris & 17 & 2,36 & 2,16 & $\mathrm{C}$ & 2,26 & & & & & \\
\hline Caranx bartholomaei & & & & & & 1 & 0,26 & 0,06 & A & $<0,01$ \\
\hline Caranx latus & 4 & 0,56 & 0,5 & Ac & 0,06 & 1 & 0,26 & 0,04 & A & $<0,01$ \\
\hline Chloroscombrus chrysurus & 19 & 2,64 & 0,35 & $\mathrm{C}$ & 0,51 & 25 & 6,60 & 3,09 & Ac & 3,73 \\
\hline Selene setapinnis & & & & & & 2 & 0,52 & 2,99 & A & 0,09 \\
\hline Selene vomer & 2 & 0,28 & 0,18 & Ac & 0,01 & 4 & 1,04 & 1,88 & Ac & 0,36 \\
\hline Trachinotus carolinus & 13 & 1,80 & 0,93 & Ac & 0,37 & 2 & 0,52 & 0,65 & Ac & 0,04 \\
\hline Trachinotus falcatus & 14 & 1,95 & 3,01 & $\mathrm{C}$ & 2,59 & 13 & 3,41 & 0,93 & $\mathrm{C}$ & 0,77 \\
\hline Trachinotus goodei & & & & & & 5 & 1,31 & 0,89 & $\mathrm{~A}$ & 0,07 \\
\hline Oligoplites $s p$. & 25 & 3,48 & 0,51 & $\mathrm{C}$ & 0,79 & & & & & \\
\hline Lutjanus synagris & 29 & 4,03 & 13,21 & $\mathrm{C}$ & 29,46 & 1 & 0,26 & 0,16 & A & $<0,01$ \\
\hline Lobotes surinamensis & & & & & & 1 & 0,26 & 0,23 & A & $<0,01$ \\
\hline
\end{tabular}


Continuação

\begin{tabular}{|c|c|c|c|c|c|c|c|c|c|c|}
\hline \multirow{2}{*}{ Espécies } & \multicolumn{5}{|c|}{ Cabuçu } & \multicolumn{5}{|c|}{ Berlinque } \\
\hline & n & $\%$ (n) & $\mathrm{p}(\%)$ & $\mathrm{C}$ & IIR & $\mathbf{n}$ & $\%$ (n) & p (\%) & $\mathrm{C}$ & IIR \\
\hline Diapterus auratus & 1 & 0,14 & 1,51 & A & 0,02 & & & & & \\
\hline Diapterus rhombeus & 29 & 4,03 & 2,08 & $\mathrm{C}$ & 3,72 & & & & & \\
\hline Eucinostomus argenteus & 6 & 0,83 & 0,79 & $\mathrm{C}$ & 0,29 & 10 & 2,62 & 0,68 & Ac & 0,32 \\
\hline Eucinostomus gula & 7 & 0,98 & 3,16 & Ac & 1,02 & 5 & 1,31 & 0,84 & Ac & 0,13 \\
\hline Eucinostomus melanopterus & 1 & 0,14 & 0,04 & $\mathrm{~A}$ & $<0,01$ & & & & & \\
\hline Conodon nobilis & 2 & 0,28 & 0,06 & A & $<0,01$ & 25 & 6,60 & 4,43 & $\mathrm{C}$ & 10,68 \\
\hline Genyatremus luteus & 2 & 0,28 & 0,29 & Ac & 0,01 & & & & & \\
\hline Pomadasys corvinaeformes & 24 & 3,34 & 2,5 & Ac & 2,73 & 23 & 6,04 & 5,79 & Ac & 6,45 \\
\hline Polydactylus virginicus & 32 & 4,45 & 1,81 & Ac & 2,67 & 86 & 22,60 & 4,17 & $\mathrm{C}$ & 34,83 \\
\hline Bairdiella ronchus & 15 & 2,08 & 2,65 & Ac & 1,83 & & & & & \\
\hline Cynoscion sp. & 7 & 0,98 & 0,24 & Ac & 0,07 & & & & & \\
\hline Larimus breviceps & 30 & 4,17 & 3,98 & $\mathrm{C}$ & 9,17 & 9 & 2,36 & 2,41 & $\mathrm{C}$ & 1,74 \\
\hline Menticirrhus littoralis & 2 & 0,28 & 0,93 & A & 0,02 & 6 & 1,60 & 2,03 & $\mathrm{C}$ & 0,78 \\
\hline Ophioscion punctatissimus & 8 & 1,11 & 0,33 & Ac & 0,12 & 48 & 12,60 & 7,24 & $\mathrm{C}$ & 22,47 \\
\hline Stellifer rastrifer & 101 & 14,04 & 0,02 & Ac & 0,02 & & & & & \\
\hline Umbrina coróides & 1 & 0,14 & 0,82 & $\mathrm{~A}$ & 0,03 & 12 & 3,14 & 1,54 & Ac & 0,89 \\
\hline Chaetodon striatus & & & & & & 1 & 0,26 & 0,14 & A & 0,01 \\
\hline Kyphosus sectatrix & & & & & & 1 & 0,26 & 0,66 & A & $<0,01$ \\
\hline Dormitator maculatus & 5 & 0,69 & 0,1 & Ac & 0,01 & & & & & \\
\hline Bathygobius soporator & 3 & 0,42 & 0,48 & Ac & 0,04 & & & & & \\
\hline Gobionellus boleosoma & 4 & 0,56 & 0,09 & Ac & 0,01 & & & & & \\
\hline Gobionellus stomatus & 3 & 0,42 & 0,32 & Ac & 0,03 & & & & & \\
\hline Microgobius meeki & 8 & 1,11 & 0,1 & Ac & 0,03 & & & & & \\
\hline Chaetodipterus faber & 46 & 6,40 & 1,55 & $\mathrm{C}$ & 7,69 & & & & & \\
\hline Acanthurus bahianus & & & & & & 1 & 0,26 & 0,16 & A & $<0,01$ \\
\hline Acanthurus chirurgus & & & & & & 1 & 0,26 & 0,16 & A & $<0,01$ \\
\hline Sphyraena guachancho & & & & & & 2 & 0,52 & 0,41 & $\mathrm{~A}$ & 0,01 \\
\hline Scomberomorus brasiliensis & 1 & 0,14 & 0,2 & A & $<0,01$ & & & & & \\
\hline Citharicthys arenaceus & 2 & 0,28 & 1,62 & Ac & 0,09 & & & & & \\
\hline Citharicthys spilopterus & 1 & 0,14 & 0,35 & A & $<0,01$ & & & & & \\
\hline Etropus crossotus & 2 & 0,28 & 0,14 & $\mathrm{~A}$ & $<0,01$ & & & & & \\
\hline Achirus lineatus & 1 & 0,14 & 0,42 & A & $<0,01$ & & & & & \\
\hline Trinectes microphthalmus & & & & & & 4 & 1,04 & 0,36 & Ac & 0,07 \\
\hline Trinectes paulistanus & 1 & 0,14 & 0,42 & A & $<0,01$ & & & & & \\
\hline Symphurus plagusia & 5 & 0,69 & 0,22 & Ac & 0,03 & 1 & 0,26 & 0,13 & A & $<0,01$ \\
\hline Symphurus trewavasae & 2 & 0,28 & 0,23 & A & $<0,01$ & & & & & \\
\hline Aluterus monocerus & 1 & 0,14 & 0,08 & A & $<0,01$ & & & & & \\
\hline Stephanolepis hispidus & 11 & 1,53 & 0,2 & Ac & 1,72 & & & & & \\
\hline Acantrostracion quadricornis & 2 & 0,27 & 5,11 & Ac & $<0,01$ & & & & & \\
\hline Sphoeroides greeleyi & 11 & 1,53 & 2,67 & $\mathrm{C}$ & 2,25 & 12 & 3,14 & 3,01 & Ac & 1,74 \\
\hline Sphoeroides testudineus & 11 & 1,53 & 10,13 & $\mathrm{C}$ & 6,86 & 8 & 2,10 & 37,9 & A & 4,88 \\
\hline Cyclichthys antillarum & 11 & 1,53 & 1,31 & $\mathrm{C}$ & 1,10 & 6 & 1,60 & 0,45 & A & 0,04 \\
\hline Total & 719 & 100 & 100 & & & 381 & 100 & 100 & & \\
\hline
\end{tabular}

$\mathrm{A}=$ acidental, $\mathrm{Ac}=$ acessória e $\mathrm{C}=$ constante 
Berlinque foram registradas 40 espécies, 32 gêneros, 21 famílias (predominando Sciaenidae, Carangidae e Engraulidae) distribuídas em 8 ordens (Tabela 1), totalizando 381 indivíduos e 2605,64g.

Houve uma maior captura de indivíduos nos meses de setembro de 2002 (18,64\% do total examinado), março $(22,95 \%)$ e julho de $2003(18,64 \%)$ na praia de Cabuçu. Em Berlinque, foi maior em janeiro (com $28,08 \%$ do total examinado) e em julho de 2003 (21,78\%). Em Cabuçu, foi registrado o maior número de espécies nos meses de novembro de 2002 e julho de 2003 (31 espécies) enquanto que em Berlinque, ocorreu em janeiro de 2003 (21 espécies) (Figura 2).

Anchoa sp., Atherinella brasiliensis (Quoy \& Gairmard, 1824), Polydactylus virginicus (Linnaeus, 1758), Larimus breviceps (Cuvier, 1830), Stellifer rastrifer (Jordan, 1889) e Chaetodipterus faber (Broussonet, 1782), representaram 42,98\% do número total de peixes coletados na praia de Cabuçu, no entanto, em Berlinque, $P$. virginicus, Ophioscion punctatissimus Meek \& Hildebrand, 1925, Anchoa sp., Chloroscombrus crhysurus (Linnaeus, 1766), Conodon nobilis (Linnaeus, 1758) e Pomadasys corvinaeformes (Steindachner, 1868) representaram $61,26 \%$ do total de peixes amostrados (Tabela 1).A maior ocorrência de indivíduos em Cabuçu, (682 indivíduos) e em Berlinque (229 indivíduos) foi registrada na estação chuvosa.

A maior captura de biomassa, em Cabuçu, foi em julho de 2002 (correspondendo a 26,64\% do total examinado) e os menores valores em janeiro de 2003 (4,70\%) (Figura 2), sendo que Lutjanus synagris (Linnaeus, 1758) e Sphoeroides testudineus (Linnaeus, 1758) apresentaram a maior biomassa contribuindo com $23,34 \%$ do total analisado. Em Berlinque, os maiores valores ocorreram em janeiro de 2003 (correspondendo a $49,85 \%$ ) e o menor em julho de $2002(6,14 \%)$, quanto $S$. testudineus contribuiu com $37,9 \%$ do total da biomassa capturada (Tabela 1). Assim, a maior produtividade em biomassa foi registrada durante a estação chuvosa em Cabuçu (1948,36g) e na estação seca, em Berlinque $(1505,5 \mathrm{~g})$.

O Índice de Importância relativa indicou que L. synagris, L. breviceps e C. faber foram espécies dominantes em Cabuçu, e $P$. virginicus, $O$. punctatissimus e C. nobilis em Berlinque (Tabela 1).

Um total de 20 espécies $(31,7 \%)$ foi de ocorrência acidental, 25 espécies foram acessórias (39,68\%) e 18 constantes $(28,57 \%)$ na comunidade analisada em Cabuçu. Destas, C. faber foi à única com $100 \%$ de ocorrência. Em Berlinque, 20 espécies (50\%) foram acidentais, 11 acessórias (27,5\%) e 9 constantes (22,5\%) (Tabela 1). No entanto, nenhuma das espécies registradas esteve presente em todas as coletas, sendo que apenas duas (5\%), P. virginicus e C. nobilis, ocorreram em 6 coletas.

Os índices de Diversidade de Shannon-Wiener (H'), Riqueza de Espécies de Margalef(RE) e Equitabilidade (E) variaram entre os meses de coleta em ambas as localidades amostradas. Em Cabuçu, os maiores valores dos índices de Shannon e Riqueza de Espécies foram registrados no mês de maio de 2003 (Figura 3a) $\left(\mathrm{H}^{\prime}=\right.$ 3,01 e $\mathrm{RE}=6,17$ - estação chuvosa), enquanto em Berlinque, ocorreu no mês de janeiro de $2003\left(\mathrm{H}^{\prime}=2,47\right.$ e $\mathrm{RE}=4,28$ - estação seca) (Figura 3b).

A análise de grupamento (Cluster) dos índices de similaridade (Bray-Curtis) estimados para as amostras temporais das 2 praias apresentou uma separação das localidades com a formação de 2 grandes grupos (grupo A - Cabuçu e grupo B - Berlinque), isolando a amostra de janeiro de 2003, na praia de Cabuçu (Figura 4a). Este resultado muito provavelmente é explicado pelo caráter atípico da amostra, quando ocorreu um reduzido número de táxons e de indivíduos. Este padrão também foi evidente na análise de ordenação (nMDS - não métrico), mostrando diferenças na composição da ictiofauna entre as duas comunidades estudadas. Os dois grupos estão conectados ao nível de $27,02 \%$ de similaridade. A similaridade entre os meses de coleta na praia de Cabuçu foi mais alta $(40,46 \%)$ do que na praia de Berlinque (33,93\%). O stress observado $(0,15)$ demonstra que as similaridades não estão adequadamente representadas pelas distâncias no plano multidimensional do mapa, embora tenha sido útil para a representação dos dados que foi corroborada pela análise de Cluster (Figura 4b). 

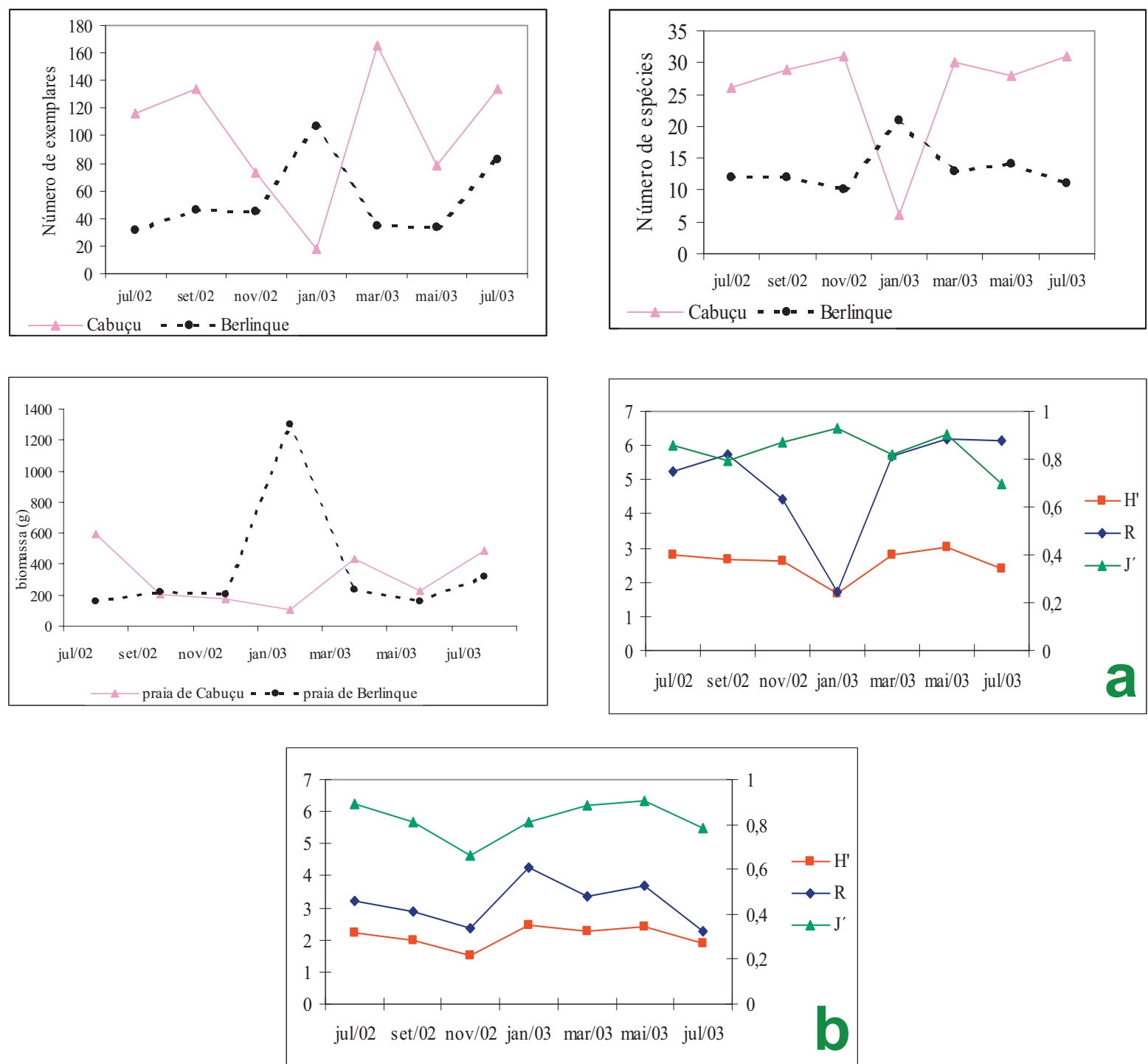

FIGURA 3: Variações mensais do número de exemplares, número de espécies, biomassa e dos índices de riqueza (R), diversidade (H') e Equitatividade (J) nas praias de Cabuçu (a) e Berlinque (b), no período de julho de 2002 a julho de 2003.

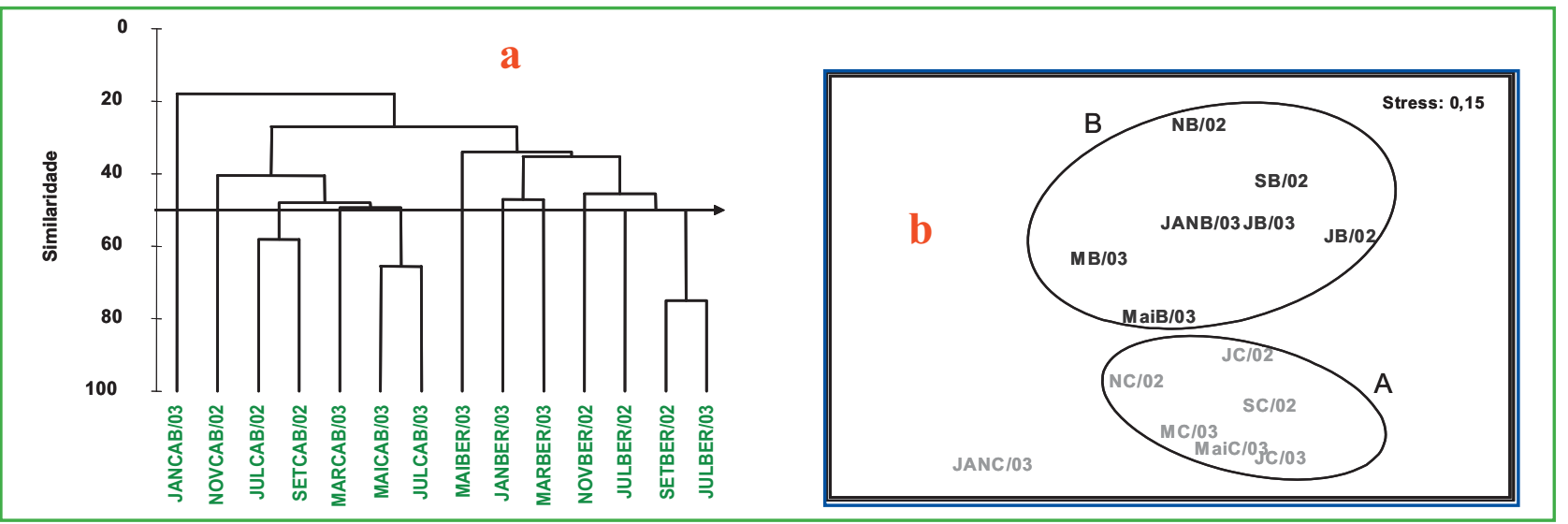

FIGURA 4: Dendrograma (a) e ordenação (b) pelo método nMDS mostrando as similaridade entre as estações de amostragem: Praias de Cabuçu e Berlinque com base na composição faunística temporal da ictiofauna registrada durante o período de Julho de 2002 a Junho de 2003 (a, Cabuçu; b, Berlinque). 
A análise de similaridade ANOSIM indicou que a composição da comunidade ictiofaunística foi significativamente diferente entre as praias de Cabuçu e Berlinque $(\mathrm{R}=0,618, \mathrm{p}=0,2 \%)$.

A praia de Cabuçu apresentou uma similaridade temporal de 40,23\% induzida pelas maiores contribuições das freqüências de ocorrências de C. faber, Anchoa sp., Oligoplites sp., Shoeroides greeleyi Gilbert, 1900, Cyclichthys antillarum (Jordan \& Rutter, 1897), M. americanus, C. chrysurus, L. synagris e Thalassophryne nattereri Steindachner, 1876 (SIMPER). Na praia de Berlinque, a similaridade foi de $40,18 \%$ devido às maiores contribuições de $P$. virginicus, $C$. nobilis, M. americanus, L. breviceps e O. punctatissimus. A dissimilaridade entre as duas praias foi $74,78 \%$ com 22 espécies responsáveis por $50,67 \%$ das contribuições (Tabela 2).

\section{Discussão}

Os dados abióticos referentes à temperatura da água, $\mathrm{pH}$ e salinidade registrada nas praias de Cabuçu e de Berlinque estão dentro da faixa de amplitude conhecida para a BTS conforme citado por Lessa et al (2001). Segundo Santos et al. (1999), na costa oeste da BTS predomina a estabilidade dos fatores ambientais com a temperatura variando em função da sazonalidade e a transparência aumentando em função da proximidade com o mar aberto; este padrão foi observado tanto na praia de Cabuçu como em Berlinque.

A abundância da ictiofauna tanto na praia de Cabuçu como de Berlinque não apresentou um padrão de variação temporal em relação ao número de exemplares e a biomassa, como observado em outras áreas costeiras estudadas (Spach et al., 2004). Segundo Araújo et al. (1997), em ambientes costeiros tropicais e subtropicais, nos quais a salinidade é relativamente estável e as variações sazonais da temperatura não são amplas, espera-se um padrão da distribuição das abundâncias menos definido relacionado com a sazonalidade.

A ictiofauna das duas praias foi caracterizada pelo domínio de formas jovens, de pequeno porte, com tamanho variando entre $10,0 \mathrm{~mm}$ e $439,0 \mathrm{~mm}$ de comprimento total, que utilizam estas praias como áreas de crescimento e alimentação, o que parece ser uma característica de ambientes de águas rasas e de margens de estuários (Pessanha et al., 2000; Santos et al., 2002; Gomes et al., 2003; Godefroid et al., 2004; Spach et al., 2004).

As famílias Sciaenidae, Carangidae, Gerreidae e Engraulidae em geral, também estão registradas como dominantes em outros estudos no Brasil (Chaves e Côrrea, 1998; Vasconcelos Filho e Oliveira, 1999; Pessanha et al., 2000; Vendel et al., 2002 e 2003;

TABELA 2: Resultado do método de similaridade de percentagem (SIMPER) para as praias de Cabuçu (Saubara) e de Berlinque (Vera Cruz), no período de julho de 2002 a julho de 2003.

\begin{tabular}{|c|c|c|c|c|}
\hline & Cabuçu & Berlinque & & \\
\hline $\begin{array}{c}\text { Similaridade média } \\
\text { dentro de cada praia (\%) }\end{array}$ & 40,23 & 40,18 & $\begin{array}{l}\text { Dissimilaridade média entre as } \\
\text { praias (\%) }\end{array}$ & 74,78 \\
\hline Chaetodipterus faber & 10,92 & - & Chaetodipterus faber & 3,86 \\
\hline Anchoa sp. & 6,58 & - & Oligoplites sp. & 2,92 \\
\hline Oligoplites sp. & 5,57 & - & Atherinella brasiliensis & 2,88 \\
\hline Sphoeroides greeleyi & 5,12 & - & Thalossophryne nattereri & 2,47 \\
\hline Cyclichthys antillarium & 4,48 & - & Cyclichthys antillarium & 2,39 \\
\hline Menticirrhus americanus & 4,48 & 8,97 & Lutjanus synagris & 2,27 \\
\hline Chloroscombrus crhysurus & 4,48 & - & Lycengraulis grossidens & 2,25 \\
\hline Lutjanus synagris & 4,45 & - & Polydactylus virginicus & 2,22 \\
\hline Thalossophryne nattereri & 4,42 & - & Ophioscion punctatissimus & 2,10 \\
\hline Polydactylus virginicus & - & 13,35 & & \\
\hline Conodon nobilis & - & 13,18 & & \\
\hline Larimus breviceps & - & 8,41 & & \\
\hline Ophioscion punctatissimus & - & 6,19 & & \\
\hline
\end{tabular}


Spach et al., 2003 e 2004). Sciaenidae, Carangidae e Engraulidae obtiveram uma maior representatividade em número de espécies em estudos reportados para outras praias arenosas ao longo da costa brasileira (Giannini e Paiva Filho, 1995; Teixeira e Almeida, 1998; Araújo e Azevedo, 2001; Godefroid et al., 2004).

A ocorrência de espécies consideradas dominantes apresentou diferenças significativas entre as áreas de estudo, porém L. breviceps, S. testudineus, Menticirrhus americanus (Linnaeus, 1758) e Lycengraulis grossidens (Agassiz, 1829) foram importantes tanto na comunidade de Cabuçu como na de Berlinque. Embora existam diferenças entre estuários com relação ao padrão de dominância das espécies, os peixes dominantes pertencem a poucos grupos taxonômicos (Santos et al., 2002) como observado nas comunidades de Cabuçu e de Berlinque.

Foram poucas as espécies que dominaram tanto em número de indivíduos quanto em peso. Este padrão assemelha-se ao de outras áreas costeiras e estuarinas do mundo e do Brasil (Monteiro-Neto et al., 1990; Teixeira e Almeida, 1998; Araújo et al., 1999; Vendel et al., 2002; Godefroid et al., 2004). Segundo Godefroid et al. (2004), este padrão reflete os diferentes estágios de crescimento em que foram capturados os exemplares uma vez que algumas espécies utilizam a área apenas para recrutamento enquanto outras a utilizam durante todo o ciclo de vida.

O grande percentual de espécies acidentais e acessórias bem como de marinhos dependentes e visitantes tanto na praia de Cabuçu como em Berlinque evidencia que as populações estão em constante renovação ao longo do ano e que poucas estão adaptadas a viverem nestes ambientes. As populações de peixes estuarinos mudam constantemente e drasticamente devido à variabilidade de gradientes ambientais, variação sazonal além dos distúrbios ambientais resultantes da atividade humana (Moyle e Cech Jr., 1996; Ramos e Vieira, 2001).

De um modo geral, a análise da comunidade de peixes realizada, através dos índices de diversidade de Shannon, Riqueza e Equitabilidade, não revelaram variações sazonais discrepantes dentro das praias estudadas, como observado por Teixeira e Almeida, (1998) e Godefroid et al. (1997). No entanto, ambas comunidades estudadas se diferenciam com relação aos índices ecológicos sendo que a praia de Cabuçu detém uma maior diversidade e riqueza. Estas diferenças são provavelmente decorrentes das condições hidrológicas e características do sedimento. A praia de Cabuçu apresenta águas calmas e elevada turbidez devido ao substrato lamoso, além do carreamento de substâncias orgânicas provenientes do processo de urbanização desse litoral, quando comparada à praia de Berlinque. Diferenças nas assembléias de peixes associadas com as características hidrológicas e substrato foram observadas por Paiva Filho e Toscano (1987), Monteiro Neto et al. (1990), Azevedo (2002) e Oliveira Neto et al. (2004).

Os grupamentos (Análise de Cluster) não evidenciaram padrões de sazonalidade, ou que reflitam diferenças na ocorrência qualitativa das espécies nas praias de Cabuçu e de Berlinque, observação também foi realizada por Godefroid et al. (1997) na praia de Pontal do Sul (Paraná, litoral sul do Brasil).

Foram obtidos baixos valores de correlação no que se refere à influência individual ou conjunta dos parâmetros abióticos sobre a estruturação do padrão biológico nas praias de Cabuçu e Berlinque. Além disso, os dados obtidos foram pontuais e não foram consideradas as variações ambientais ao longo do dia e, portanto, os parâmetros ambientais utilizados neste estudo não são considerados bons descritores das comunidades estudadas.

A dinâmica da ocupação dos ambientes estuarinos, lagunares e de baías por juvenis como área de proteção e alimentação bem como área de residência para espécies que completam seu ciclo de vida, além de servir como áreas de passagem para as espécies diádromas ou ocasionalmente usadas por visitantes marinhos e de água doce (Amanieu e Lasserre, 1982, apud Azevedo, 2002). Assim as assembléias de peixes das praias de Cabuçu e Berlinque podem ser consideradas variáveis no tempo e constituídas ao acaso.

\section{Agradecimentos}

Aos estagiários do Laboratório de Ictiologia da UEFS pelo auxílio nas coletas de campo. Ao biólogo Leonardo E. de Moraes pelo auxílio nas coletas. Este 
trabalho foi integralmente financiado pela Universidade Estadual de Feira de Santana.

\section{Referências}

Araújo, F. G.; Azevedo, M. C. C 2001. Assemblages of SoutheastSouth Brazilian coastal systems base on the distribution of fishes. Estuarine, Coastal and Shelf Science, 52: 729-738.

Araújo, F. G.; Bailey, R. G.; Williams, W. P. 1999. Spatial and temporal variations in fish populations in the upper Thames estuary. Journal of Fish Biology, 55: 836-853.

Araújo, F. G.; Cruz Filho, A. G.; Azevedo, M. C. C.; Santos, A. C. A. ; Fernandes, L. A. M. 1997. Estrutura da comunidade de peixes jovens da margem continental da baía de Sepetiba, RJ. Acta Biológica Leopoldensia, 19 (1): 61-83.

Azevedo, M. C. C. 2002. Peixes demersais da Baía de Sepetiba, RJ: Distintas assembléias ao longo de um gradiente ambiental Tese de Doutorado, Universidade Federal Rural do Rio de Janeiro, Brasil, 99pp.

Beaumord, A. C. 1991. As comunidades de peixes do rio Manso. Chapada dos Guimarães, MT: uma abordagem ecológica numérica. Dissertação de Mestrado, Instituto de Biociências Carlos Chagas, Brasil, 108pp.

Chaves, P. T. C.; Côrrea, M. F. M. 1998. Composição ictiofaunística da área de manguezal da baía de Guaratuba, Paraná, Brasil. Revista Brasileira de Zoologia, 15 (1): 195-202.

Clarke, K. R.; Warwick, R. W. 2001. Change in marine communities: An aproach to statistical analysis and interpretation. $2^{\text {nd }}$ ed. PRIMER- E, Plymouth, UK, 165pp.

Dajoz, R. 1973. Ecologia Geral. $2^{a}$ ed. Ed. Vozes/EDUSP, São Paulo, Brasil , 472pp.

Falcón, G. (ed.). 1997. Baía de Todos os Santos: Diagnóstico sócioambiental e subsídios para a gestão. Gérmen/UFBA-NIMA, Salvador, Brasil, 244pp.

Giannini, R.; Paiva Filho, A. M. 1995. Análise comparativa da ictiofauna da zona de arrebentação de praias arenosas do estado de São Paulo, Brasil. Boletim do Instituto Oceanográfico, 43 (2): 141152.

Gomes, M. P.; Cunha, M. S.; Zalmon, I. R. 2003. Spatial and temporal variations of diurnal ichthyofauna on surf-zone of São Francisco do Itabapoana beaches, Rio de Janeiro state, Brazil. Brazilian Archives of Biology and Technology, 46 (4): 653-664.

Godefroid, R. S.; Hofstaetter, M.; Spach, H. L. 1997. Structure of the fish assemblage in the surf zone beach at Pontal do Sul, Paraná. Nerítica, 11: 77-93.

Godefroid, R. S.; Spach, H. L.; Santos, C.; MacLaren, G.; Schwarz Jr., R. 2004. Mudanças temporais na abundância e diversidade da fauna de peixes do infralitoral raso de uma praia, sul do Brasil. Iheringia Série Zoológica, 94 (1): 95-104.

Lessa, G.C., Dominguez, J.M.L., Bittencourt, A.C.S.P. and Brichta, A. 2001. The tides and tidal circulation of Todos os Santos Bay, Northeast Brazil: a general characterization. An. Acad. Bras. Cienc. 73.2: 245-261.

Monteiro-Neto, C.; Blacher, C.; Laurent, A. A. S.; Snizek, F. N.; Canozzi, M. B.; Tabajara, L. L. C. de A. 1990. Estrutura da comunidade de peixes em águas rasas na região de Laguna, Santa Catarina, Brasil. Atlântica, 12 (2): 53-69.

Moyle, P. B.; Cech Jr., J. J. 1996. Fishes: an introduction to ichthyology. $3^{\text {rd }}$ ed. New Jersey Prentice Hall, New Jersey, USA, 590pp.

Oliveira Neto, J. F.; Godefroid, R. S.; Queiroz, G. M. N.; Schwarz Jr., R. 2004. Variação diuturna na captura de peixes em uma planície de maré da baía de Paranguá, PR. Acta Biológica Leopoldensia, 26 (1): $125-138$.

Paiva Filho, A. M.; Toscano, A. P. 1987. Estudo comparativo e variação sazonal da ictiofauna na zona entremarés do mar Casado - Guarujá e mar Pequeno - São Vicente, SP. Boletim do Instituto Oceanográfico, 35 (2): 153-165.

Pessanha, A. L. M.; Araújo, F. G.; Azevedo, M. C. C.; Gomes, I. D. 2000. Variações temporais e espaciais na composição e estrutura da comunidade de peixes jovens da baía de Sepetiba, Rio de Janeiro. Revista Brasileira de Zoologia, 17 (1): 251-261.

Ramos, L. A.; Vieira, J. P. 2001. Composição específica e abundância de peixes de zonas rasas dos cinco estuários do Rio Grande do Sul, Brasil. Boletim do Instituto de Pesca, 27 (1): 109-121.

Rodriguez-Romero, J.; Abitia-Cárdenas, L. A.; Galván-Magaña, F.; Chávez-Ramos, H. 1994. Composicion, abundancia y riqueza especifica de la ictiofauna de Bahia Conception, Baja Califórnia sur, México. Ciências Marinas, 20 (3): 321-350.

Santos, A. C. de A.; Castellucci, F. R.; Nepomuceno, C. F.; Santos, E. P.; Sena, M. P. 1999. Distribuição e recrutamento do peixe-rei Xenomelaniris brasiliensis (Osteichthyes, Atherinidae) na margem continental oeste da Baía de Todos os Santos, Bahia, Brasil. Acta Biológica Leopoldensia, 21 (1): 107-118.

Santos, C.; Schwarz Jr., R.; Oliveira Neto, J. F.; Spach, H. L. 2002. A ictiofauna em duas planícies de maré do setor euhalino da baía de Paranaguá, PR. Boletim do Instituto de Pesca, 28 (1): 49-60.

Santos, F. L. B. 2001. Levantamento da ictiofauna do estuário do rio Formoso (Pernambuco, Brasil) através da pesca de camboa. Dissertação de Mestrado. Universidade Federal de Pernambuco, Brasil, 76pp.

Spach, H. L.; Godefroid, R. S.; Santos, C.; Schwarz Jr., R.; Queiroz, G. M. L. 2004. Temporal variation in fish assemblage composition on a tidal flat. Brazilian Journal of Oceanography, 52 (1): 47-58.

Spach, H. L.; Santos, C.; Godefroid, R. S. 2003. Padrões temporais na assembléia de peixes na gamboa do Sucuriú, Baía de Paranaguá, Brasil. Revista Brasileira de Zoologia, 20 (4): 591-600.

Teixeira, R. L.; Almeida, G. I. 1998. Composição da ictiofauna de três praias arenosas de Maceió, $\mathrm{AL}$ - Brasil. Bolletim do Museu de Biologia Mello Leitão (Nova Série), 8: 21-38.

Vasconcelos Filho, A.L.; Oliveira, A. M. E. 1999. Composição e ecologia da ictiofauna do canal de Santa Cruz (Itamaracá - PE - Brasil). Trabalhos Oceanográficos da Universidade Federal de Pernambuco, 27 (1): 101-113.

Vendel, A. L.; Spach, H. L.; Lopes, S. G.; Santos, C. 2002. Structure and dynamics of fish assemblages in a tidal creek environment. Brazilian Archives of Biology and Tecnology, 45 (3): 365-373.

Vendel, A. L.; Lopes, S. G.; Santos, C.; Spach, H. L. 2003. Fish assemblages in a tidal flat. Brazilian Archives of Biology and Tecnology, 46 (2): 233-242.

Zar, J. H. 1999. Biostatiscal analysis. $4^{\text {rd }}$ ed. Printice Hall International, New York, USA, 662pp. 